\title{
A New Formulation Approach for Location-Routing Problems
}

\author{
Hunkar Toyoglu • Oya Ekin Karasan • Bahar Yetis Kara
}

Received: 26 April 2011 / Accepted: 19 December 2011 / Published online: 12 January 2012 (C) Springer Science+Business Media, LLC 2012

\begin{abstract}
A Location-Routing Problem (LRP) combines two difficult problems, facility location and vehicle routing, and as such it is inherently hard to solve. In this paper, we propose a different formulation approach than the common arc-based product-flow (Arc-BPF) approach in the literature. We associate product amounts to the nodes of the network resulting in a node-based product-flow (Node-BPF) formulation. Our main objective is to develop LRP models with fewer constraints and variables, which can be solved more efficiently. To introduce the proposed approach, we reformulate a complex four-index Arc-BPF LRP model from the literature as a three-index Node-BPF model, which computationally outperforms the former. We then introduce a heuristic method.
\end{abstract}

Keywords Location-routing problem • Mixed integer linear programming • Logistics • Network design

\section{Introduction}

Organizations in today's complex environments, whether civilian organizations in competitive markets or military organizations in hostile war arenas, need to operate at their full potential to survive. In order to achieve this potential, they must make proper strategic decisions that affect the longterm direction of the entire organization, tactical decisions that focus on

H. Toyoglu $(\varangle)$ · O. E. Karasan · B. Y. Kara

Department of Industrial Engineering, Bilkent University,

Ankara 06800, Turkey

e-mail: hunkar@bilkent.edu.tr 
intermediate-term issues, and operational decisions that concentrate on dayto-day activities within the organization.

In the special case of transportation organizations, Crainic and Laporte (1997) identify these decision levels, review the literature, and provide the common mathematical models from an operations research perspective. According to them, strategic decisions include the design of the physical network and the location of main facilities. They refer to these issues as Logistics System Design which, consists of location, network design, and regional multimodal planning models. Tactical decisions concern mainly the route choice, service type, etc. They categorize these issues into two groups of which the first is long distance (less-than-truckload or rail) and the second is short distance (several pick up and deliveries mainly by truck) transportation. They refer to the former as Service Network Design and to the latter as Vehicle Routing Problems (VRP). In daily planning, operational decisions are taken in a dynamic environment, where time is an important factor (e.g. vehicle or crew schedules, time windows), and involve uncertain factors, such as demand. Therefore, they investigate these issues under two headings, dynamic and stochastic models (mainly VRP models).

Strategic, tactical and operational problems can be collectively identified as Distribution Network Design Problems (DNDP). Within the context of DNDP the Location-Routing Problem (LRP) merges facility location and vehicle routing into a single problem where strategic location and tactical/operational routing decisions are taken simultaneously. Salhi and Rand (1989) evaluate the effect of ignoring routing when locating facilities and clearly show that separating facility location from vehicle routing may lead to suboptimal decisions. This interdependence between the location of facilities and vehicle routing necessitates the combination of such decisions (the number and location of the facilities and the routes emanating from the facilities to serve multiple demand points), which in turn leads to LRPs.

The LRP has been studied since the early 1970s and there are several surveys on it (see, e.g., Berman et al. 1995; Laporte 1988; List et al. 1991; Min et al. 1998; Ahipasaoglu et al. (2004, unpublished); Nagy and Salhi 2007). According to the recent classification of Toyoglu et al. (2011) the majority of the LRP literature considers delivery or pickup of a single product, uses deterministic and hypothetical data, includes two layers and locates uncapacitated multiple facilities at one layer with a discrete solution space, utilizes a capacitated homogeneous vehicle fleet, considers a single planning period with no time restrictions, has a single objective function, allows a customer to be supplied by only a single vehicle, incorporates no inventory and uses heuristic methods for solution.

The LRP accepts many different formulations, of which the most widely used are vehicle-flow and commodity-flow formulations (see, e.g., Laporte 1988), where the latter explicitly considers the quantity of commodities traveling in the system whereas the former only considers the vehicle circulation. Complex LRP models (see, e.g., Hansen et al. 1994; Yi and Ozdamar 2007) in the literature generally use commodity-flow formulation, since it enables the 
inclusion of more details and facilitates modeling of real world applications. For example, one of the most realistic LRP models is due to Ambrosino and Scutellà (2005) where they model a four-layer (plants, central depots, regional depots, and customers) LRP with inventory considerations and introduce dynamic version of the model.

In general, the LRP is NP-hard (see, e.g., Laporte 1988; Min et al. 1998; Nagy and Salhi 2007). Although there exist several exact solution methodologies for LRPs (see, e.g., Laporte and Nobert 1981; Laporte et al. 1986, 1988; Laporte and Dejax 1989; Belenguer et al. 2011; Baldacci et al. 2011; Karaoglan et al. 2011), almost all reviews or surveys urge the use of heuristics and a significant amount of studies resort to heuristics due to the complexity of LRPs.

Recently, Nagy and Salhi (2007) classify LRP heuristics into four groups, namely; sequential, clustering-based, iterative, and hierarchical methods. In general, all methods decompose an LRP into its major components, which is location, allocation, and routing. They then solve these parts either repeatedly, iteratively, or simultaneously. In particular, sequential methods (see, e.g., Or and Pierskalla 1979; Nambiar et al. 1989; Srivastava and Benton 1990) usually first solve a location problem to decide which depots to open and how to allocate customers to open depots. Then, given the locations of the open depots a vehicle routing problem is solved. Clustering-based methods (see, e.g., Billionnet et al. 2005; Schwardt and Dethloff 2005; Barreto et al. 2007) first group the customers into clusters such that each cluster contains one potential depot or vehicle. Then, for each cluster a VRP is solved either after or before locating a depot. Iterative methods (see, e.g., Perl and Daskin 1985; Salhi and Fraser 1996; Wu et al. 2002; Prins et al. 2007; Duhamel et al. 2010; TavakkoliMoghaddam et al. 2010) usually construct two or more subproblems each one including one or two of the major components. Then, these subproblems are solved repeatedly such that a subproblem provides some input to the next subproblem in an iterative manner. Hierarchical methods (see, e.g., Nagy and Salhi 1996; Albareda-Sambola et al. 2005; Melechovsky et al. 2005; Bozkaya et al. 2010) treat the location subproblem as the main problem and the routing subproblem as the subordinate problem that is embedded into the main problem. A hierarchical method then solves the location problem while in each step of the location problem it solves a routing problem which in turn provides information to the location problem.

Our main objective in this study is to provide a modeling approach different from the common ones existing in the literature. To the authors' knowledge, this is the first reported study in the operational research literature to provide such a mathematical formulation for modeling LRPs. To introduce our modeling approach, we consider the variant of the state of the art LRP model defined by Toyoglu et al. (2011). Their LRP model, in contrast with the majority of the LRP literature, utilizes a capacitated heterogeneous vehicle fleet, considers capacitated facilities, has three layers, includes two-sided time windows, locates facilities at two different layers, distributes multiple products and allows multiple sourcing. 
The main difference between the study of Toyoglu et al. (2011) and this study is the attempt to reduce the number of constraints and variables in the mathematical model by applying a new node-based formulation approach. The foremost idea is to reduce the solution time purely by modeling, and therefore the contribution of this paper lies in its mathematical formulation.

The remainder of this study is structured as follows. We define the problem in Section 2. We introduce our modeling approach and develop a mathematical model in Section 3. We compare the computational performance of our model with previous results in the literature in Section 4. We introduce a heuristic solution methodology in Section 5 and test it in Section 6. We conclude with Section 7.

\section{Problem definition}

Toyoglu et al. (2011) propose a replenishment system which is called Mobile Ammunition Distribution System (Mobile-ADS) where ammunition (ammo) flows from depots to combat units via transfer points. Ammo that is produced or procured is first received by main depots from where it is moved forward with rail network by trains to Fixed Transfer Points (Fixed-TPs). The flow from the main depots to Fixed-TPs is not included in the Mobile-ADS model. It is assumed that in case of war there will be enough ammo at the main depots and current rail network structure and equipment are sufficient to carry the demand to the Fixed-TPs on time.

From Fixed-TPs ammo is moved to Mobile Transfer Points (Mobile-TPs) by commercial trucks on road networks. Then Mobile-TPs issue ammo to their attached combat units with special ammo trucks which have the capability to

Fig. 1 Mobile Ammo Distribution System on the battlefield

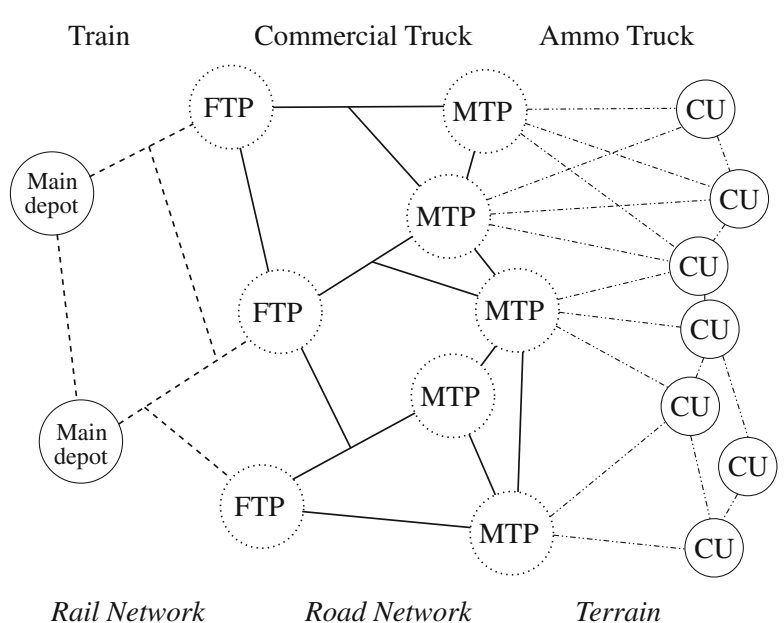

Rail Network

Terrain 
move on terrain. A Mobile-ADS on the battlefield is presented in Fig. 1. In this figure FTP, MTP and CU stands for Fixed-TP, Mobile-TP, and combat unit respectively.

In other words, ammo is transferred from trains to commercial trucks and then to ammo trucks at Fixed-TPs and Mobile-TPs. Assuming the locations of the main depots and combat units are known in advance, the remaining decisions that must be made are; (1) the locations of Fixed-TPs and MobileTPs and (2) the routes and schedules of commercial and ammo trucks to distribute ammo among Fixed-TPs, Mobile-TPs, and combat units. MobileADS design problem is an LRP since it contains both location and routing problems.

\section{Mathematical formulation}

Customarily, LRP models involve a decision variable such as $f_{i j v}$ which denotes the quantity of product traveled on arc $(i, j)$ with vehicle $v$. Multi-product models add another index to differentiate between commodities. Henceforth, we refer to this approach as arc-based product-flow (Arc-BPF).

In this study we investigate a different product-flow approach to develop an LRP model with fewer number of constraints and variables. Briefly, we replace $f_{i j v}$ with two new decision variables, where $f_{-}$out $_{i v}$ denotes the quantity of product that is sent from node $i$ and $f_{-} i n_{j v}$ denotes the quantity of product that is dropped to node $j$ with vehicle $v$. Henceforth, we refer to this approach as node-based product-flow (Node-BPF). To the authors' knowledge, no model that incorporates Node-BPF approach has previously been reported in the LRP literature.

We consider the Mobile-ADS in Fig. 1 to be a directed and connected network $G=(N, A)$ that is defined by a set $N$ of nodes and a set $A$ of arcs. Three types of nodes (Fixed-TPs, Mobile-TPs and combat units), and two types of vehicles (commercial and ammo trucks) exist. The model specifications are given in Table 1.

\subsection{Product flow balance constraints}

The product flow decision variables can be seen in Fig. 2. Note that in $f_{t p \_o u t} t_{i v p}$ and $m t p \_i n_{i v p}, v$ is a commercial truck, whereas in $m t p \_o u t_{i v p}$ and $c u \_i n_{i v p}, v$ is an ammo truck.

$$
\sum_{v \in V_{M}} c u \_i n_{i v p}=Q_{i p} \quad \forall i \in N_{C}, p \in P
$$


Table 1 Model specifications

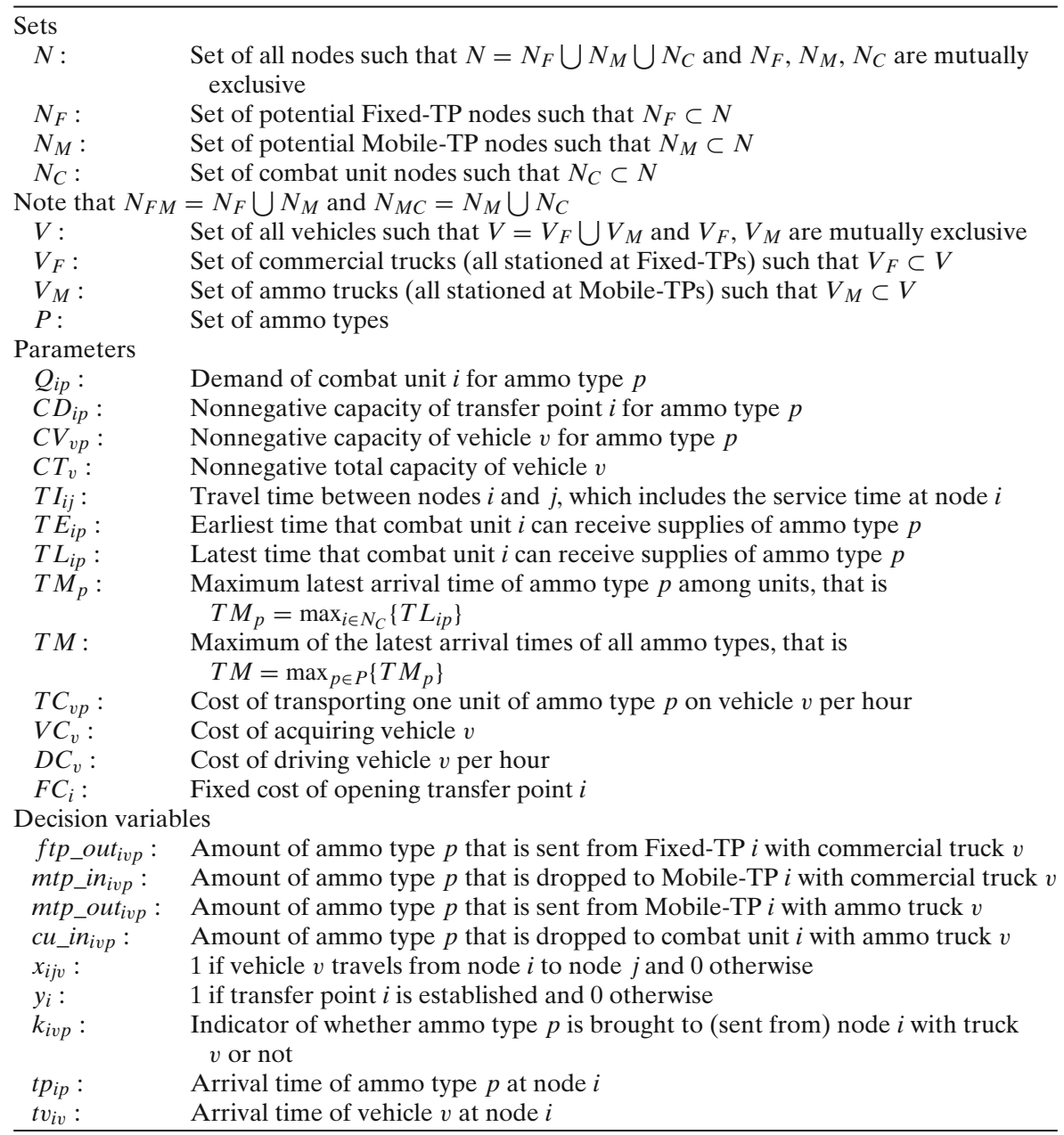

Constraints (1) ensure that demand of a combat unit for each ammo type must be satisfied by ammo trucks.

$$
\begin{aligned}
& \sum_{i \in N_{F}} f t p \_o u t_{i v p}=\sum_{i \in N_{M}} m t p \_i n_{i v p} \quad \forall v \in V_{F}, p \in P \\
& \sum_{i \in N_{M}} m t p \_o u t_{i v p}=\sum_{i \in N_{C}} c u \_i i_{i v p} \quad \forall v \in V_{M}, p \in P
\end{aligned}
$$

Fig. 2 Node-based product-flow decision variables
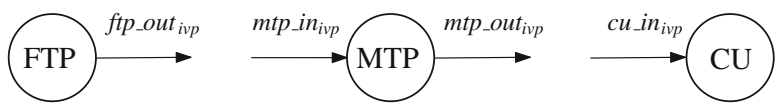

Ammo Trucks 


$$
\sum_{v \in V_{F}} m t p \_i n_{i v p}=\sum_{v \in V_{M}} m t p \_o u t_{i v p} \quad \forall i \in N_{M}, p \in P
$$

Constraints (2) guarantee that each commercial (ammo) truck drops its entire load, which it loads from a Fixed-TP (Mobile-TP), to Mobile-TPs (combat units). Constraints (3) ensure that total inflow of an ammo type to a MobileTP that is dropped by commercial trucks is equal to the total outflow of that ammo type from that Mobile-TP that is sent by ammo trucks.

3.2 Vehicle flow balance constraints

$$
\begin{array}{ll}
\sum_{i \in N_{F}} \sum_{j \in N_{M}} x_{i j v} \leq 1 & \forall v \in V_{F} \\
\sum_{i \in N_{M}} \sum_{j \in N_{C}} x_{i j v} \leq 1 & \forall v \in V_{M}
\end{array}
$$

Constraints (4) indicate that a vehicle can be dispatched from a transfer point at most once.

$$
\begin{array}{ll}
\sum_{j \in N_{M}} x_{j i v}=\sum_{j \in N_{M}} x_{i j v} \quad \forall i \in N_{F}, v \in V_{F} \\
\sum_{j \in N_{C}} x_{j i v}=\sum_{j \in N_{C}} x_{i j v} \quad \forall i \in N_{M}, v \in V_{M} \\
\sum_{\substack{j \in N_{F M} \\
j \neq i}} x_{j i v}=\sum_{\substack{j \in N_{F M} \\
j \neq i}} x_{i j v} \quad \forall i \in N_{M}, v \in V_{F} \\
\sum_{\substack{j \in N_{M C} \\
j \neq i}} x_{j i v}=\sum_{\substack{j \in N_{M C} \\
j \neq i}} x_{i j v} \quad \forall i \in N_{C}, v \in V_{M}
\end{array}
$$

Constraints (5) force each vehicle to turn back to its home transfer point where it is allocated. Constraints (6) require that each vehicle leaves the node that it enters.

\subsection{Capacity constraints}

$$
\begin{array}{ll}
\sum_{v \in V_{F}} f_{t p \_o u t} t_{i v p} \leq C D_{i p} \cdot y_{i} & \forall i \in N_{F}, p \in P \\
\sum_{v \in V_{M}} m t p \_o u t_{i v p} \leq C D_{i p} \cdot y_{i} & \forall i \in N_{M}, p \in P
\end{array}
$$


Constraints (7) ensure that the amount of ammo type $p$ that is transferred from a transfer point cannot exceed the appropriate capacity. They also guarantee that there is no flow from/through any closed transfer point.

$$
\begin{gathered}
\sum_{i \in N_{M}} m t p \_i n_{i v p} \leq C V_{v p} \cdot \sum_{i \in N_{F}} \sum_{j \in N_{M}} x_{i j v} \quad \forall v \in V_{F}, p \in P \\
\sum_{i \in N_{C}} c u \_i n_{i v p} \leq C V_{v p} \cdot \sum_{i \in N_{M}} \sum_{j \in N_{C}} x_{i j v} \quad \forall v \in V_{M}, p \in P \\
\sum_{p \in P} \sum_{i \in N_{F}} f t p \_o u t_{i v p} \leq C T_{v} \cdot \sum_{i \in N_{F}} \sum_{j \in N_{M}} x_{i j v} \quad \forall v \in V_{F} \\
\sum_{p \in P} \sum_{i \in N_{M}} m t p \_o u t_{i v p} \leq C T_{v} \cdot \sum_{i \in N_{M}} \sum_{j \in N_{C}} x_{i j v} \quad \forall v \in V_{M}
\end{gathered}
$$

Constraints (8) ensure that vehicle capacities are not exceeded and forbid unused vehicles to carry any flow. All vehicles also have total capacity restrictions respected by constraints (9).

$$
\begin{aligned}
& f t p \_o u t_{i v p} \leq C V_{v p} \cdot \sum_{j \in N_{M}} x_{i j v} \forall i \in N_{F}, v \in V_{F}, p \in P \\
& m t p \_i n_{i v p} \leq C V_{v p} \cdot \sum_{\substack{j \in N_{F M} \\
j \neq i}} x_{j i v} \quad \forall i \in N_{M}, v \in V_{F}, p \in P \\
& \text { mtp_out }_{i v p} \leq C V_{v p} \cdot \sum_{j \in N_{C}} x_{i j v} \quad \forall i \in N_{M}, v \in V_{M}, p \in P \\
& \text { cu_in ivp } \leq C V_{v p} \cdot \sum_{\substack{j \in N_{M C} \\
j \neq i}} x_{j i v} \quad \forall i \in N_{C}, v \in V_{M}, p \in P
\end{aligned}
$$

Constraints (10) state that if there is an outflow (inflow) of an ammo type from (to) a node by a vehicle then that vehicle must be dispatched from (enter to) that node. Conversely, they maintain that if no vehicle is dispatched from (enters to) a node then no outflow (inflow) of any ammo type can exit from (enter to) that node.

\subsection{Relation constraints}

We use the binary decision variable $k_{i v p}$ as an indicator of (1) whether ammo type $p$ is brought to Mobile-TP (combat unit) $i$ with commercial (ammo) truck $v$ or not and (2) whether ammo type $p$ is sent from Mobile-TP $i$ with ammo truck $v$ or not. Mathematically, (1) $k_{i v p}=1$ if $m t p_{-} i n_{i v p}>0$ for all $i \in N_{M}, v \in V_{F}, p \in P$ or $k_{i v p}=0$ otherwise, (2) $k_{i v p}=1$ if $m t p \_o u t_{i v p}>0$ for 
all $i \in N_{M}, v \in V_{M}, p \in P$ or $k_{i v p}=0$ otherwise, (3) $k_{i v p}=1$ if $c u \_i n_{i v p}>0$ for all $i \in N_{C}, v \in V_{M}, p \in P$ or $k_{i v p}=0$ otherwise.

$$
\begin{gathered}
k_{i v p} \leq m t p \_i n_{i v p} \quad \forall i \in N_{M}, v \in V_{F}, p \in P \\
k_{i v p} \leq m t p \_o u t_{i v p} \quad \forall i \in N_{M}, v \in V_{M}, p \in P \\
k_{i v p} \leq c u \_i n_{i v p} \quad \forall i \in N_{C}, v \in V_{M}, p \in P \\
m t p \_i n_{i v p} \leq C V_{v p} \cdot k_{i v p} \quad \forall i \in N_{M}, v \in V_{F}, p \in P \\
m t p \_o u t_{i v p} \leq C V_{v p} \cdot k_{i v p} \quad \forall i \in N_{M}, v \in V_{M}, p \in P \\
c u \_i n_{i v p} \leq C V_{v p} \cdot k_{i v p} \quad \forall i \in N_{C}, v \in V_{M}, p \in P
\end{gathered}
$$

Constraints (11) and (12) set the correct logical relationships between the decision variables $k$ and $m t p \_i n, m t p \_o u t, c u \_i n$. They maintain that if a vehicle drops (carries) an ammo type to (from) a node then there must exit some inflow (outflow) of that ammo type to (from) that node with that vehicle. Conversely, they also ensure that if a vehicle does not drop (carry) an ammo type to (from) a node then there cannot exit any inflow (outflow) of that ammo type to (from) that node with that vehicle.

\subsection{Time related constraints}

$$
\begin{aligned}
& T E_{i p} \leq t p_{i p} \leq T L_{i p} \quad \forall i \in N_{C}, p \in P \\
& t v_{i v}+T I_{i j} \cdot x_{i j v}-T M \cdot\left(1-x_{i j v}\right) \leq t v_{j v} \quad \forall i \in N_{F}, j \in N_{M}, v \in V_{F} \\
& \forall i, j \in N_{M}, i \neq j, v \in V_{F} \\
& \forall i \in N_{M}, j \in N_{C}, v \in V_{M} \\
& \forall i, j \in N_{C}, i \neq j, v \in V_{M}
\end{aligned}
$$

Constraints (13) impose the time window requirements of combat units on the model for all ammo types. Constraints (14) are the classical subtour elimination constraints of Miller et al. (1960).

$$
\begin{array}{ll}
t p_{i p}-T M_{p} \cdot\left(1-k_{i v p}\right) \leq t v_{i v} & \forall i \in N_{M}, v \in V_{M}, p \in P \\
t v_{i v}-T M_{p} \cdot\left(1-k_{i v p}\right) \leq t p_{i p} & \forall i \in N_{M}, v \in V_{F}, p \in P \\
& \forall i \in N_{C}, v \in V_{M}, p \in P
\end{array}
$$

Constraints (15) and (16) set the correct relationships between the arrival times of vehicles and products to a node. They force that the arrival time of an ammo type at a Mobile-TP (combat unit) is exactly the arrival time of each 
commercial (ammo) truck carrying that product to that transfer point (combat unit).

Note that time window constraints refer to the latest ammo arrival and ensure that the latest delivery is within the allowed time windows. In other words, arrival time of ammo type $p$ at a node is in fact the time of the arrival of the latest vehicle carrying that ammo type to that node.

\subsection{Objective function}

In this study, we consider the costs of transfer point establishment and vehicle acquisition plus the cost of truck driving jointly in the objective function.

$$
\begin{aligned}
\min z= & \sum_{i \in N_{F M}} F C_{i} \cdot y_{i} \\
& +\sum_{i \in N_{F}} \sum_{j \in N_{M}} \sum_{v \in V_{F}} V C_{v} \cdot x_{i j v}+\sum_{i \in N_{M}} \sum_{j \in N_{C}} \sum_{v \in V_{M}} V C_{v} \cdot x_{i j v} \\
& +\sum_{i \in N} \sum_{j \in N} \sum_{v \in V} D C_{v} \cdot T I_{i j} \cdot x_{i j v} .
\end{aligned}
$$

Equation (17) is the total fixed cost of opening transfer points, Eq. (18) is the total acquisition cost of used trucks, and finally Eq. (19) is the total driving cost of the trucks.

\subsection{Valid inequalities}

In order to improve the behavior of the model just presented, we included the following valid inequalities (20) in the formulation.

$$
\begin{aligned}
\sum_{j \in N_{M}} x_{i j v} \leq \sum_{p \in P} f t p \_o u t_{i v p} \quad \forall i \in N_{F}, v \in V_{F} \\
\sum_{j \in N_{C}} x_{i j v} \leq \sum_{p \in P} m t p \_o u t_{i v p} \quad \forall i \in N_{M}, v \in V_{M} \\
\sum_{\substack{j \in N_{F M} \\
j \neq i}} x_{j i v} \leq \sum_{p \in P} m t p \_i n_{i v p} \quad \forall i \in N_{M}, v \in V_{F} \\
\sum_{\substack{j \in N_{M C} \\
j \neq i}} x_{j i v} \leq \sum_{p \in P} c u \_i n_{i v p} \quad \forall i \in N_{C}, v \in V_{M}
\end{aligned}
$$

Constraints (20) maintain that if there is no outflow (inflow) with a vehicle from (to) a node then that vehicle must not be dispatched from (enter to) that node. Conversely, they guarantee that if a vehicle is dispatched from (enters to) a node then some outflow (inflow) of an ammo type with that vehicle must exist from (to) that node. 
Table 2 Details of the nine test problem instances with $|N|=31$

\begin{tabular}{lccccccccc}
\hline & PI A & PI B & PI C & PI D & PI E & PI F & PI G & PI H & PI I \\
\hline$|P|$ & 3 & 3 & 3 & 4 & 4 & 4 & 5 & 5 & 5 \\
$|V|$ & 24 & 36 & 36 & 24 & 36 & 36 & 24 & 36 & 36 \\
Truck usage & $75 \%$ & $50 \%$ & $83 \%$ & $75 \%$ & $50 \%$ & $83 \%$ & $75 \%$ & $50 \%$ & $83 \%$ \\
\hline
\end{tabular}

\section{Computational experiments with the Node-BPF model}

In this section, we use the same nine test problems described in Toyoglu et al. (2011) to compare the performances of the proposed models. All computations are conducted on a computer with $2.4 \mathrm{GHz}$ CPU, 4 GB RAM and Windows XP operating system. We use CPLEX 9.1 as the solver and GAMS 22.0 as the modeling language.

In the test problems, 3 potential Fixed-TPs, 8 potential Mobile-TPs and 20 combat units are considered. 3 different ammo collections $(|P|=3,4,5)$ and 3 different levels of truck usage percentages $(75 \%, 50 \%, 83 \%)$ are considered. At the $75 \%$ level, there are 8 commercial and 16 ammo trucks, of which at least 6 commercial and 12 ammo trucks must be used. At the $50 \%$ level, there are 12 commercial and 24 ammo trucks, of which at least 6 commercial and 12 ammo trucks must be used. At the $83 \%$ level, there are 12 commercial and 24 ammo trucks, of which at least 10 commercial and 20 ammo trucks must be used. The minimum number of trucks are imposed on the model simply by adjusting the capacity of the vehicles in the input data. For example, at the $50 \%$ level capacity of a commercial (ammo) truck is 20 (10) tons, whereas it is 13 (7) tons at the $83 \%$ level.

Details of the problem instances can be seen in Table 2, where $|N|,|P|$ and $|V|$ are the numbers of nodes, ammo types, and vehicles, respectively.

We first compare the Node-BPF and Arc-BPF models based on the total number of the decision variables and constraints. Table 3 presents some general statistics on the model sizes that have been obtained from the Model Statistics section of GAMS 22.0. Note that the Node-BPF model has $70 \%$

Table 3 General statistics of the Node-BPF and Arc-BPF models

\begin{tabular}{|c|c|c|c|c|c|c|c|c|}
\hline & \multicolumn{2}{|c|}{$\begin{array}{l}\text { Non zero } \\
\text { elements }\end{array}$} & \multicolumn{2}{|c|}{ Equations } & \multicolumn{2}{|c|}{ Variables } & \multicolumn{2}{|c|}{$\begin{array}{l}\text { Discrete } \\
\text { variables }\end{array}$} \\
\hline & $\begin{array}{l}\text { Arc } \\
\text { BPF }\end{array}$ & $\begin{array}{l}\text { Node } \\
\text { BPF }\end{array}$ & $\begin{array}{l}\text { Arc } \\
\text { BPF }\end{array}$ & $\begin{array}{l}\text { Node } \\
\text { BPF }\end{array}$ & $\begin{array}{l}\text { Arc } \\
\text { BPF }\end{array}$ & $\begin{array}{l}\text { Node } \\
\text { BPF }\end{array}$ & $\begin{array}{l}\text { Arc } \\
\text { BPF }\end{array}$ & $\begin{array}{l}\text { Node } \\
\text { BPF } \\
\end{array}$ \\
\hline PI A & 240,513 & 80,593 & 27,996 & 10,704 & 81,493 & 26,105 & 11,623 & 11,659 \\
\hline PI B-C & 358,217 & 120,797 & 40,596 & 15,916 & 121,257 & 39,105 & 16,679 & 17,483 \\
\hline PI D & 306,767 & 91,263 & 34,402 & 13,002 & 104,464 & 27,184 & 12,123 & 12,171 \\
\hline PI E-F & 456,751 & 136,775 & 49,754 & 19,322 & 155,388 & 40,708 & 17,179 & 18,251 \\
\hline PI G & 373,021 & 101,933 & 40,808 & 15,300 & 127,435 & 28,263 & 12,623 & 12,683 \\
\hline PI H-I & 555,285 & 152,753 & 58,912 & 22,728 & 189,519 & 42,311 & 17,679 & 19,019 \\
\hline Average & 381,759 & 114,019 & 42,078 & 16,162 & 129,926 & 33,946 & 14,651 & 15,201 \\
\hline
\end{tabular}


Table 4 Computational results of the Node-BPF and Arc-BPF models

\begin{tabular}{|c|c|c|c|c|c|c|c|c|}
\hline & & Gaps & after & & & & & \\
\hline & & $5 \mathrm{~m}$ & $15 \mathrm{~m}$ & $1 \mathrm{~h}$ & $2 \mathrm{~h}$ & $4 \mathrm{~h}$ & $11 \mathrm{~h}$ & $14 \mathrm{~h}$ \\
\hline PI A & Arc-BPF & - & - & - & - & - & 9.11 & 6.71 \\
\hline PI B & & - & - & - & - & - & 8.74 & 8.74 \\
\hline Other PIs & & - & - & - & - & - & - & - \\
\hline PI A & Node-BPF & - & 4.58 & 1.42 & 1.40 & 1.37 & 1.35 & 1.35 \\
\hline PI B & & - & - & 6.95 & 1.44 & 1.44 & 1.43 & 1.43 \\
\hline PI C & & - & - & - & - & - & - & - \\
\hline PI D & & - & - & - & 7.27 & 4.32 & 1.42 & 1.42 \\
\hline PI E & & 12.00 & 11.89 & 6.88 & 1.50 & 1.44 & 1.42 & 1.40 \\
\hline PI F & & - & - & - & - & - & - & - \\
\hline PI G & & - & - & - & - & - & - & 15.64 \\
\hline PI H & & - & - & - & - & 15.71 & 11.22 & 11.22 \\
\hline PI I & & - & - & - & - & - & - & - \\
\hline
\end{tabular}

fewer non zero elements, $62 \%$ fewer equations and $73 \%$ fewer variables but only $3 \%$ more discrete variables than Arc-BPF model has.

We then compare the models according to the optimality gap, that is reported by CPLEX (the gap between the best integer objective and the objective of the best node remaining), reached in a certain time period. Table 4 exhibits the corresponding computational results. We use strong branching for selecting the branching variable, best-estimate search for selecting the next node when backtracking and CPLEX's default settings for the other parameters. Computations for a problem are terminated after $14 \mathrm{~h}$. Abbreviations m and $\mathrm{h}$ stand for minutes and hours, and dashes indicate that no feasible solution can be found within the associated run-time. It can be seen in the table that the reduction in the number of the equations and variables of the Node-BPF model when compared with those of the Arc-BPF model has paid off in terms of expanding the set of problem instances where a solution can be recovered. In addition, the Node-BPF model has offered significant improvements to the optimality gaps of the instances for which Arc-BPF model was able to obtain solutions in $14 \mathrm{~h}$. Specifically, Node-BPF model improves the optimality gap of PI A from 6.71 to $1.35 \%$ and PI B from 8.74 to $1.43 \%$.

Even as such, the current NP-hard LRP model is still computationally unattractive due to its solution time when considered as a decision support tool during the short term tactical decision making process in distributing ammo on the battlefield. Ultimately, we need to develop a heuristic method, which forms the subject of the following section.

\section{Solution methodology}

In this section, we present a "VRP first-LRP second" type heuristic to solve the Mobile-ADS design problem. Broadly speaking, it can be said that our method falls under the route first, location-allocation second category of Min et al. (1998). 
In addition, the solution approach proposed in this study is a clusteringbased heuristic according to the categorization of Nagy and Salhi (2007). In general terms, we first partition all combat units into some clusters such that for each cluster there is at least one potential Mobile-TP site. Then, we solve at least one VRP for each cluster and using the solutions of VRPs we solve an LRP for the rest of the problem. The details of the heuristic are as follows.

"VRP first-LRP second" heuristic consists of three phases. Phase 1 is the clustering part that partitions the combat units into clusters. Phase 2 is the VRP part that finds the routes of ammo trucks distributing ammo from MobileTPs to combat units in each cluster. Phase 3 is the LRP part that decides on the locations of the transfer points to open and the routes of commercial trucks distributing ammo from Fixed-TPs to Mobile-TPs. The flowchart of this method can be seen in Fig. 3.

\section{Phase 1. Clustering}

In this phase, we group all combat units into clusters such that each cluster is reachable by a potential Mobile-TP which can serve the total demand of units within the specified time windows.

(a) Step 1. Form the clusters

Due to the military nature of the Mobile-ADS problem, we need to consider some military requirements when forming the clusters. In real life each combat unit (battalion in this case) belongs to a distinct brigade which readily forms a cluster. Furthermore, because of the manpower and equipment limitations each brigade (cluster in this case) opens a single Mobile-TP and the combat units of that cluster can be served by only that transfer point. Hence, we need to state that Mobile-ADS problem definition inherently includes clusters such that each brigade is a cluster with at least one potential Mobile-TP. If we had not had these clusters, we should have used some clustering technique (see, e.g., Bruns and Klose 1995; Min 1996; Barreto et al. 2007 for LRPs and Solomon 1986, 1987; Braysy and Gendreau 2005 for VRPs with time windows) to construct them. Now, let $K$ be the cluster set and proceed to Step 2.

(b) Step 2. Modify ammo truck costs

Each cluster has at least one potential Mobile-TP meaning that it may have two or more. Note that in the next phase a VRP will be solved for each potential Mobile-TP within each cluster. While solving VRPs the ammo truck set should be arranged wisely otherwise it can easily lead to infeasibility when in fact the problem is feasible. Hence, we modify the acquisition costs of ammo trucks slightly such that every truck has a different cost. With the modified costs, each VRP of a cluster will start to use the ammo trucks starting from the least expensive one. Hence, Mobile-TPs of the same cluster will use the same ammo trucks that eventually will prevent the unnecessary reservation of more than enough trucks. Proceed to Step 3. 


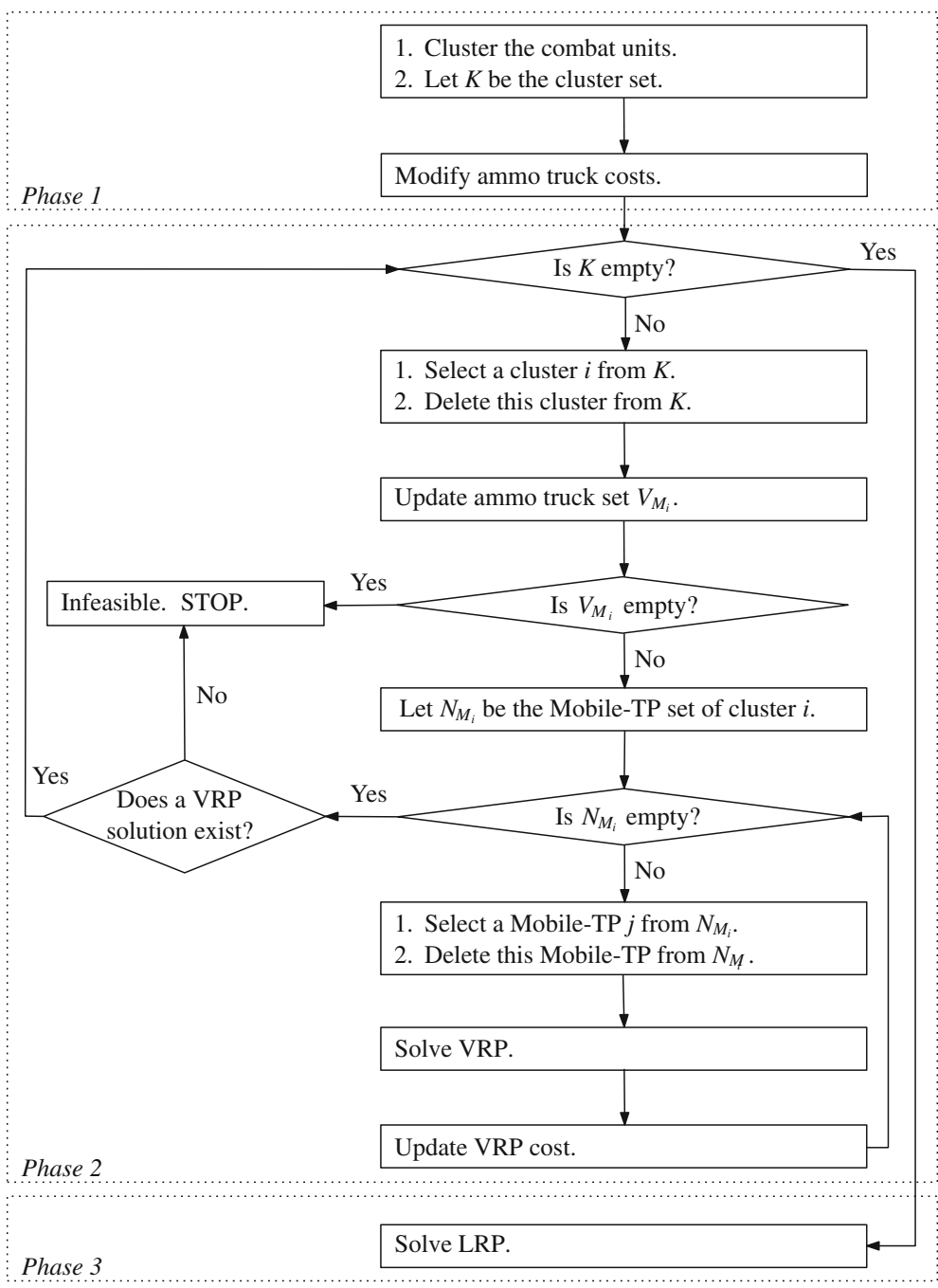

Fig. 3 Flowchart of the heuristic

2. Phase 2. Vehicle routing problem (VRP)

In this phase, we solve a VRP for each potential Mobile-TP.

(a) Step 3. Select a cluster

If set $K$ is empty this means that all clusters have been processed already, and we are ready to proceed to the next phase, hence go to Step 10. Otherwise, select a cluster, $i$, remove it from $K$, and proceed to Step 4.

(b) Step 4. Update ammo truck set $\left(V_{M}\right)$

In this step, we try to increase the computational efficiency. To do so we make an additional (but not unrealistic) assumption that all 
combat units require less than truck loads. Hence, the number of trucks that are used by any Mobile-TP can be bounded above by the number of units of its cluster. We employ the following procedure. Let $V_{M_{i}}$ represent the modified ammo truck set that will be used by the potential Mobile-TPs of cluster $i$. We modify the ammo truck set as follows and proceed to Step 5.

let $V_{M_{i}}=\emptyset$

for $\left(v \in V_{M}\right)$ do

while $\left(\left|V_{M_{i}}\right| \leq \mid\right.$ cluster $\left.i \mid\right)$ do

if $v \notin \cap_{k \in K: i \neq k} V_{M_{k}}$

end while

let $v \in V_{M_{i}}$

end for.

(c) Step 5. Check infeasibility

If set $V_{M_{i}}$ is empty this means that there is no unused ammo truck left for that cluster to dispatch and the problem is infeasible, hence STOP. Otherwise, proceed to Step 6.

(d) Step 6. Select a potential Mobile-TP

Let $N_{M_{i}}$ be the set of potential Mobile-TPs of cluster $i$. If set $N_{M_{i}}$ is empty this means that a VRP for all potential Mobile-TPs of that cluster is already solved and nothing is remained to be processed, hence proceed to Step 7. Otherwise, select a Mobile-TP, $j^{*}$, delete it from $N_{M_{i}}$ and go to Step 8.

(e) Step 7. Check infeasibility

If no VRP has a feasible solution this means that the demands of the units of this cluster cannot be satisfied in the given problem setting according to the specified constraints. In other words, the problem is infeasible, hence STOP. Otherwise, if at least one feasible solution exists for the VRP of a Mobile-TP, then this means that we processed all potential Mobile-TPs of this cluster. We are then ready to process a new cluster, hence go to Step 3.

(f) Step 8. Solve VRP

In this step, we solve a VRP including the combat units of cluster $i$ and Mobile-TP $j^{*}$ using the vehicle set $V_{M_{i}}$. Let $N_{C_{i}}$ be the set of all combat units of cluster $i$ and $N_{C_{i}}^{*}=N_{C_{i}} \cup j^{*}$. The VRP to be solved in this step is presented below.

$$
\begin{aligned}
\min o=\sum_{g \in N_{C_{i}}} \sum_{v \in V_{M_{i}}} V C_{v} \cdot x_{j^{*} g v} \\
\quad+\sum_{g \in N_{C_{i}}^{*}} \sum_{\substack{h \in N_{C_{i}}^{*} \\
h \neq g}} \sum_{v \in V_{M_{i}}} D C_{v p} \cdot T I_{g h} \cdot x_{g h v}
\end{aligned}
$$

s.t.

(1), (2b), (4b), (5b), (6b), (7b), (8b), (9b), (10c), (10d)

(11b), (11c), (12b), (12c), (13), (14), (15), (16), (20b), (20d) 


$$
\begin{aligned}
t p_{j^{*} p} & \geq \max _{g \in N_{F}}\left\{T I_{g j^{*}}\right\} \quad \forall p \in P \\
k_{j^{*} v p} & \leq \sum_{g \in N_{C_{i}}} x_{j^{*} g v} \quad \forall v \in V_{M_{i}}, p \in P \\
k_{g v p} & \leq \sum_{\substack{j \in N_{C_{i}}^{*} \\
j \neq g}} x_{j g v} \quad \forall g \in N_{C_{i}}, v \in V_{M_{i}}, p \in P \\
\sum_{v \in V_{M_{i}}} k_{g v p} & \geq 1 \quad \forall g \in N_{C_{i}}, p \in P
\end{aligned}
$$

Note that in this step we solve a VRP for a particular Mobile-TP $j^{*}$ which is considered open. Therefore, decision variable $y_{i}$ and all operations over the set $N_{M}$ disappear and above constraints, which are not given explicitly, should be adjusted accordingly.

Note also that at this phase, we still do not know which Fixed-TP to be opened. To maintain time window restrictions, we introduce constraints (21) which assure that the arrival time of any ammo at Mobile-TP $j^{*}$ is not less than the maximum of the traveling times between $j^{*}$ and the potential Fixed-TPs. Hence, no matter which Fixed-TP is opened in Phase 3, the arrival time of any ammo to any opened Mobile-TP will always be greater than or equal to the traveling time in between. To further reduce the solution time, we use valid inequalities (22), (23) and (24) which we test and find useful through extensive computational experiments. Valid inequalities (22) and (23) require that if a vehicle drop (take) an ammo type to (from) a node then that node must be on that vehicle's route. Valid inequalities (24) maintain that the demand of each combat unit for each ammo type must be satisfied by at least one ammo truck.

(g) Step 9. Update VRP cost

A careful examination reveals two differences between the objective value of the VRP in the heuristic (denoted by $o$ ) and that of the real model (denoted by $z$ ). The first difference is the fixed opening costs of Mobile-TPs. $z$ includes these costs but $o$ does not, since there is only one transfer point in each VRP of Step 8 and this transfer point is already considered open. The second difference is the vehicle acquisition costs, which are modified in Step 2. Therefore, to get the real total cost and to compare it with the cost of the original formulation, we need to modify the VRP costs as follows, $\bar{o}=o+$ $F C_{j^{*}}+\sum_{g \in N_{C_{i}}} \sum_{v \in V_{M_{i}}} \varepsilon_{v} \cdot x_{j^{*} g v}$, where $\varepsilon_{v}$ represents the difference between the actual and modified costs of ammo truck $v$.

Since the VRP phase of Mobile-TP $j^{*}$ is completed, go to Step 6.

3. Phase 3. Location routing problem (LRP)

In the first two phases, we determine the routes and schedules of ammo trucks that distribute ammo from each Mobile-TP to the units of the cluster 
where its home Mobile-TP belongs. In this phase, we will be interested in the distribution network design and the distribution from Fixed-TPs to Mobile-TPs using commercial trucks. By distribution network design, we mean that we are going to decide which Mobile-TPs and Fixed-TPs to open. Since, both location and routing decisions exist in this phase, we will solve an LRP model including all Fixed-TPs and Mobile-TPs.

(a) Step 10. Solve LRP

In this step we solve a two layer LRP in which Fixed-TPs lie on the first and Mobile-TPs lie on the second layer. We need to decide (1) which Fixed-TP and Mobile-TP (one for each cluster) to open and (2) routes of the commercial trucks among open transfer points. The LRP to be solved in this step is shown below.

$$
\begin{aligned}
\min s=\sum_{i \in N_{F}} F C_{i} \cdot y_{i} & +\sum_{i \in N_{F}} \sum_{j \in N_{M}} \sum_{v \in V_{F}} V C_{v} \cdot x_{i j v} \\
& +\sum_{i \in N_{F}} \sum_{j \in N_{F M}} \sum_{v \in V_{F}} D C_{v} \cdot T I_{i j} \cdot x_{i j v}+\sum_{i \in N_{M}} \bar{o}_{i} \cdot y_{i}
\end{aligned}
$$

s.t.

(2a), (4a), (5a), (6a), (7a), (8a), (9a), (10a), (10b)

(10c), (10d), (11a), (12a), (13), (20a), (20b), (20c)

(14) $\forall i \in N_{F}, j \in N_{M}, v \in V_{F} ; \forall i, j \in N_{M}, i \neq j, v \in V_{F}$

(16) $\forall i \in N_{M}, v \in V_{F}, p \in P$

$\sum_{v \in V_{F}} m t p \_i n_{i v p}=\sum_{j \in N_{C_{i}}} Q_{j p} \cdot y_{i} \quad \forall i \in N_{M}, p \in P$

$\sum_{j \in N_{M_{i}}} y_{j}=1 \quad \forall i \in K$

$t p_{i p} \leq \min _{g \in N_{C_{i}}}\left\{T L_{g p}-T I_{i g}\right\} \quad \forall i \in N_{M}, p \in P$

$k_{i v p} \leq \sum_{\substack{j \in N_{F M} \\ j \neq i}} x_{j i v} \quad \forall i \in N_{M}, v \in V_{F}, p \in P$

$\sum_{i \in N_{F}} y_{i} \geq\left\lceil\frac{\sum_{p \in P} \sum_{i \in N_{C}} Q_{i p}}{\max _{p \in P, i \in N_{F}}\left\{C D_{i p}\right\}}\right\rceil$

$$
\sum_{\substack{j \in N_{F M} \\ j \neq i}} x_{i j v} \leq y_{i} \quad \forall i \in N_{M}, v \in V_{F}
$$

Let $\bar{o}_{j}$ represent the $\bar{o}$ of Mobile-TP $j$. In detail, $\bar{o}_{j}$ is the sum of the fixed cost of opening Mobile-TP $j$, the acquisition cost of the ammo trucks that are used by Mobile-TP $j$ and the distribution cost of 
ammo to combat units from Mobile-TP $j$. By appending $\bar{o}_{j}$ into above objective function, we incorporate all previous VRPs into the LRP as a cost parameter.

Constraints (25) maintain the correct flow of ammo types to MobileTPs. Constraints (26) make sure that there is one open Mobile-TP per cluster. Constraints (27) set the upper limit for arriving times of ammo types at Mobile-TPs.

As we do in solving VRPs, we use the following valid inequalities for solving the LRP to help reduce the solution time. Valid inequalities (28) require that if a vehicle drop (take) an ammo type to (from) a node then that node must be on that vehicle's route. Valid inequalities (29) set the lower bound for the number of transfer points to be opened. Valid inequalities (30) provide that no vehicle can be dispatched from or pass through a closed transfer point

\section{Computational experiments with the heuristic method}

In this section, we evaluate the performance of our heuristic empirically by applying it to the nine problem instances described in Toyoglu et al. (2011). We then present a larger scale application and investigate whether the heuristic can produce feasible solutions within reasonable solution times.

1. The Benchmark Instances

Recall that, with the heuristic we solve a VRP for each Mobile-TP and using the solutions of these VRPs we solve a single LRP for the rest of the problem. To calculate the run times of the heuristic we introduce the following rule.

(a) We solve each VRP by CPLEX 9.1. either to optimality or it is terminated if it cannot improve the best objective function value for $30 \mathrm{~s}$.

(b) After running all VRPs, we solve the single LRP by using the following methodology and by CPLEX 9.1. We separate problem instances into two groups regarding the results of the Node-BPF model run results. In detail, PI A, B, D, E, G and $\mathrm{H}$ are in the first group since Node-BPF model finds a feasible solution in $14 \mathrm{~h}$. Nevertheless, it cannot find a feasible solution to PI C, F and I in the same time limit and hence these problem instances constitute the second group. For the LRP runs, we aim for a target value and run the model until it reaches that target. For the first group, we use the objective function values that are attained after $14 \mathrm{~h}$ as benchmarks. That is, we run the LRP until it reaches the objective function value that is obtained by the Node-BPF model in $14 \mathrm{~h}$. For example, in PI A the termination criteria for the LRP is to reach an objective function value that is smaller or equal to 925.44. Since no objective function value for the second group exists, we aim for a target optimality gap for this group. 
Table 5 Run times (seconds) of the heuristic

\begin{tabular}{|c|c|c|c|c|c|c|c|c|c|}
\hline & PI A & PI B & PI C & PI D & PI E & PIF & PI G & PI H & PI I \\
\hline VRPs & 281 & 282 & 325 & 324 & 328 & 437 & 510 & 403 & 527 \\
\hline LRP & 18 & 434 & 129 & 25 & 324 & 16 & 13 & 87 & 2,297 \\
\hline Total & 299 & 716 & 454 & 349 & 652 & 453 & 523 & 490 & 2,824 \\
\hline
\end{tabular}

We request the heuristic to obtain an objective value within at most $10 \%$ of the optimum. We calculate the optimality gap of the heuristic by using the best bound obtained with the Node-BPF model after $14 \mathrm{~h}$. For example, since we seek an optimality gap of $10 \%$ the stopping criteria for the LRP in PI C is to reach an objective value of 1342.29, which is calculated as $0.1=\frac{x-1208.06}{x} \Rightarrow x=1342.29$

(c) Total run time of the heuristic is the sum of the run times of all VRPs and that of the single LRP.

Table 5 demonstrates the run times of the heuristic for each problem instance. For example, in PI A all eight VRPs take 281 s to run in total. The single LRP runs for $18 \mathrm{~s}$ before it reaches an objective value which is equal to or smaller than that of the Node-BPF model. Briefly, the run time of the heuristic is $299 \mathrm{~s}$ in PI A. Run times of the heuristic range from 5 to $47 \mathrm{~min}$, averaging to $13 \mathrm{~min}$.

Table 6 compares the performances of the Node-BPF and heuristic model. To better test the performance of the heuristic, we present the optimality gap of the heuristic calculated by using the best bound obtained with the Node-BPF model after $14 \mathrm{~h}$. For example, for PI B $\frac{926.23-913.01}{926.23} \times 100=$ 1.43 and $\frac{925.98-913.01}{925.98} \times 100=1.40$. Dashes indicate that no feasible solution can be obtained in $14 \mathrm{~h}$. The table reveals that on the average the heuristic attains a better objective function value in 13 min than that of the Node$\mathrm{BPF}$ model in $14 \mathrm{~h}$. In addition, the heuristic finds a feasible solution within $10 \%$ of the optimum in about 8 to 47 min even in the problem instances PI $\mathrm{C}, \mathrm{F}$ and I where Node-BPF model cannot find a feasible solution in $14 \mathrm{~h}$. It is worth noting that not only the Node-BPF model but also the Arc-BPF model would also benefit from the proposed clustering-based heuristic.

Table 6 Computational results of the Node-BPF model and the heuristic

\begin{tabular}{|c|c|c|c|c|c|c|c|}
\hline & \multicolumn{4}{|c|}{ Node-BPF model } & \multicolumn{3}{|c|}{ Heuristic } \\
\hline & Time & Best bound & Best integer & Gap (\%) & Time & Objective & Gap (\%) \\
\hline PI A & $14 \mathrm{~h}$ & 912.93 & 925.44 & 1.35 & $299 \mathrm{~s}$ & 925.38 & 1.35 \\
\hline PI B & $14 \mathrm{~h}$ & 913.01 & 926.23 & 1.43 & $716 \mathrm{~s}$ & 925.98 & 1.40 \\
\hline PI C & $14 \mathrm{~h}$ & 1208.06 & - & - & $454 \mathrm{~s}$ & 1340.71 & 9.89 \\
\hline PI D & $14 \mathrm{~h}$ & 912.55 & 925.65 & 1.42 & $349 \mathrm{~s}$ & 925.64 & 1.42 \\
\hline PI E & $14 \mathrm{~h}$ & 912.66 & 925.65 & 1.40 & $652 \mathrm{~s}$ & 925.42 & 1.38 \\
\hline PI F & $14 \mathrm{~h}$ & 1207.50 & - & - & $453 \mathrm{~s}$ & 1338.95 & 9.82 \\
\hline PI G & $14 \mathrm{~h}$ & 912.82 & 1082.03 & 15.64 & $523 \mathrm{~s}$ & 1076.84 & 15.23 \\
\hline PI H & $14 \mathrm{~h}$ & 912.54 & 1027.86 & 11.22 & $490 \mathrm{~s}$ & 981.36 & 7.01 \\
\hline PI I & $14 \mathrm{~h}$ & 1207.29 & - & - & $2824 \mathrm{~s}$ & 1341.04 & 9.97 \\
\hline
\end{tabular}




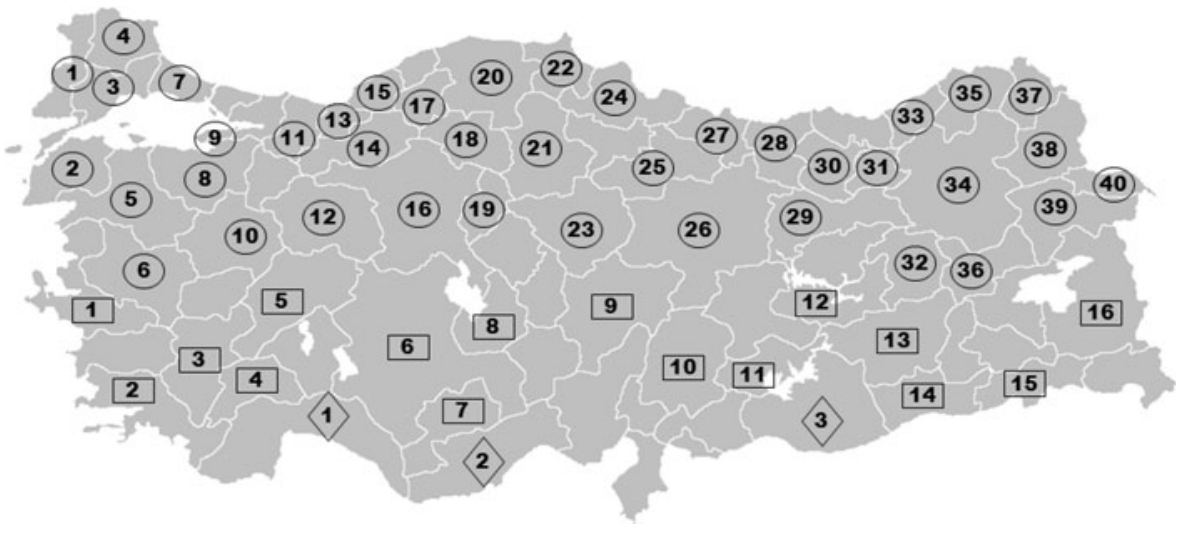

Fig. 4 Layout of the large scenario

2. Large Problem Instances

To better test the performance of our heuristic, we implement it on a larger scale scenario that describes a distribution design over the cities of Turkey. In addition, we also want to show that direct extensions of our formulation could be applied to a wide variety of other distribution or logistics scenarios other than military. Figure 4 illustrates the layout of the scenario, where circles represent known locations of the customers, whereas rectangles and diamonds represent potential locations of MobileTPs and Fixed-TPs.

Details of the problem instances are provided in Table 7. As can be seen in Fig. 4 there are 40 customers, 16 potential Mobile-TP and 3 potential Fixed-TP locations that sums up to 59 nodes in total. Problem instances are created by considering different numbers of products to be distributed and of trucks available. In all instances at least $75 \%$ of the available trucks should be used to satisfy the demands.

There are two factors that need to be taken into account in running the heuristic, namely solution quality and time. Mobile-ADS model can be used as a decision support tool to design the distribution network both in a daily base and in an ad hoc environment. The two situations differ from each other in the required time for an answer. Ad hoc situations are unplanned contingencies that occur during the day where an immediate solution is required, whereas in regular daily planning relatively long time

Table 7 Details of the larger problem instances with $|N|=59$

\begin{tabular}{|c|c|c|c|c|c|c|c|c|c|}
\hline & PI J & PI K & PI L & PI M & PI N & PI O & PI P & PI Q & $\overline{\text { PI R }}$ \\
\hline$P \mid$ & 3 & 3 & 3 & 4 & 4 & 4 & 5 & 5 & 5 \\
\hline$|V|$ & 24 & 36 & 48 & 24 & 36 & 48 & 24 & 36 & 48 \\
\hline
\end{tabular}


Table 8 Computational results of the heuristic

\begin{tabular}{llrrrr}
\hline & \multicolumn{2}{l}{ Emphasis quality } & & \multicolumn{2}{c}{ Emphasis time } \\
\cline { 2 - 3 } \cline { 6 - 6 } & Time & Objective & & Time & Objective \\
\hline PI J & $5770 \mathrm{~s}$ & 931.59 & $227 \mathrm{~s}$ & 934.05 \\
PI K & $7485 \mathrm{~s}$ & 1243.69 & & $1524 \mathrm{~s}$ & 1256.00 \\
PI L & $7477 \mathrm{~s}$ & 1248.44 & & $1253 \mathrm{~s}$ & 1252.30 \\
PI M & $6418 \mathrm{~s}$ & 932.93 & & $1056 \mathrm{~s}$ & 936.08 \\
PI N & $7629 \mathrm{~s}$ & 1247.56 & & $1399 \mathrm{~s}$ & 1251.88 \\
PI O & $8190 \mathrm{~s}$ & 1251.03 & & $2196 \mathrm{~s}$ & 1305.08 \\
PI P & $5943 \mathrm{~s}$ & 934.55 & & $1424 \mathrm{~s}$ & 987.12 \\
PI Q & $6925 \mathrm{~s}$ & 1246.97 & & $3116 \mathrm{~s}$ & 1252.79 \\
PI R & $6134 \mathrm{~s}$ & 1283.89 & $2617 \mathrm{~s}$ & 1307.00 \\
\hline
\end{tabular}

is available. To cater for both conditions we design two different heuristic run methodologies.

(a) Emphasize quality over time: For daily planning requirements. Run all VRPs and the LRP until each one reaches to an optimality gap of $1 \%$ or terminate if they can not improve the best objective function value for $3 \mathrm{~min}$.

(b) Emphasize time over quality: For unplanned contingencies. Run all VRPs and the LRP until each one reaches to an optimality gap of $3 \%$ or terminate if they can not improve the best objective function value for $1 \mathrm{~min}$.

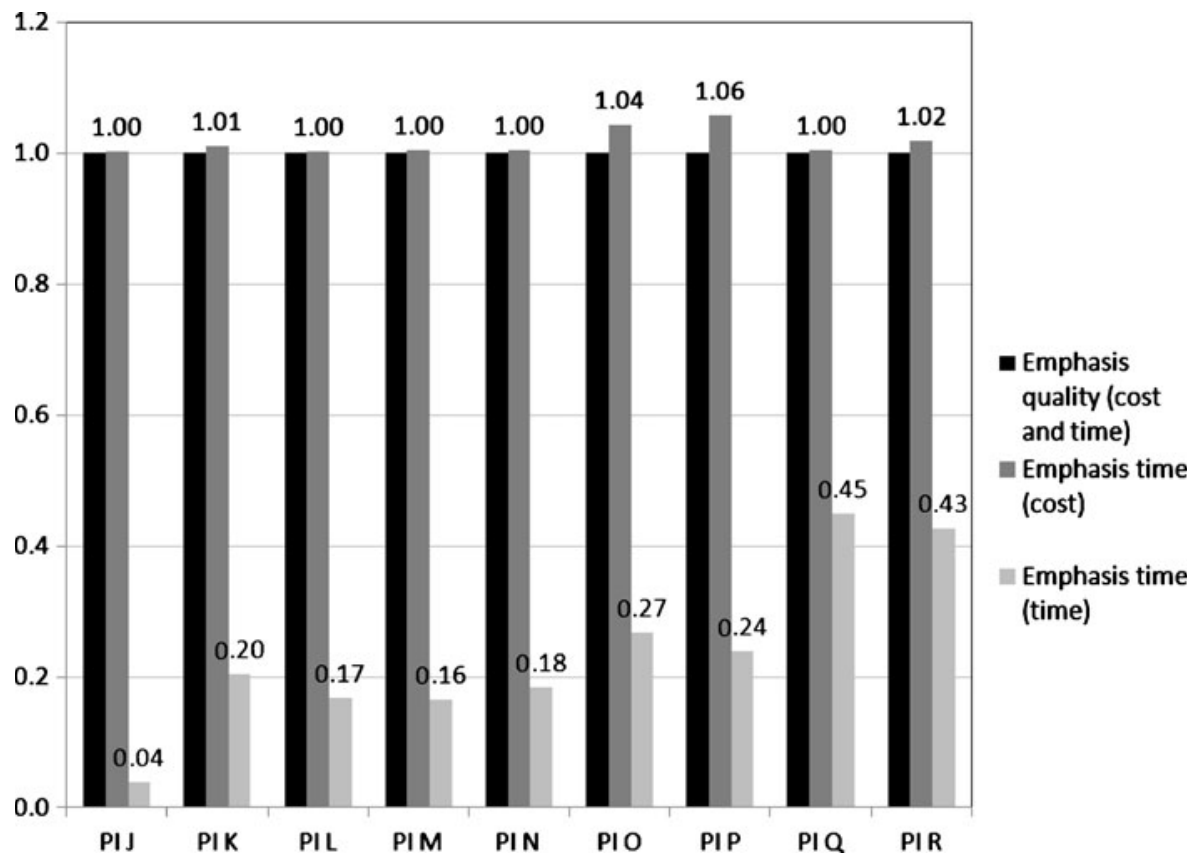

Fig. 5 Comparison of the heuristic run methodologies 


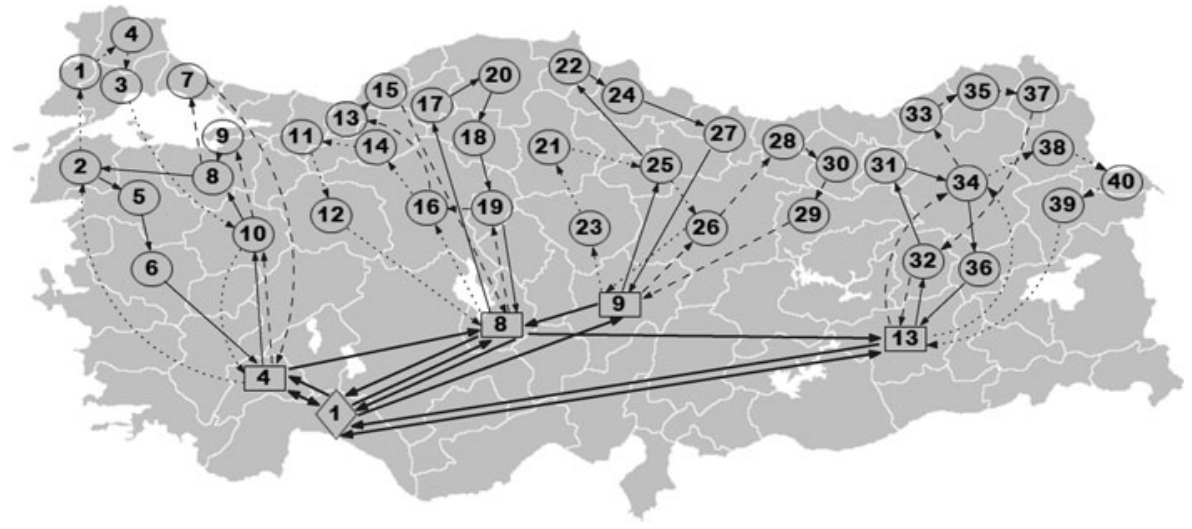

Fig. 6 Solution of the problem instance $\mathrm{J}$

Table 8 displays the heuristic run results of both run methodologies. When comparing the run methodologies, we base the comparison on their objective function values as well as the solution times. The first methodology finds an answer for each instance in about $2 \mathrm{~h}$, whereas the solution times of the second methodology range from a minimum of about 4 min to a maximum of about $52 \mathrm{~min}$, the average being about $27 \mathrm{~min}$.

A comparison of the heuristic results is shown in Fig. 5. Not surprisingly, the first methodology obtains lower objective function values (higher quality solutions) since it runs longer. However, computational results show that with the second run methodology it is possible to get slightly higher costs with much lower solution times. For example, in PI K the second methodology reaches a cost only $1 \%$ higher than that of the first methodology with $80 \%$ less solution time. Briefly, the second methodology provides $76 \%$ reduction in solution times on the average, at the cost of approximately $2 \%$ increase in objective function values.

We present the solution of PI J by emphasizing quality in Fig. 6 as an example. Thick lines between the Fixed-TPs and Mobile-TPs represent commercial truck tours, whereas thin lines between the Mobile-TPs and combat units represent the tours of the ammo trucks.

Solution quality or solution time, which one receives more attention depends on the situation. Anyhow it is easy to address such trade offs between solution time and cost with the proposed heuristic, by adjusting the stopping criteria of VRPs and of the LRP accordingly.

\section{Conclusions}

In this study, we present a different commodity-flow LRP formulation where product-flow is considered on the nodes of the network, and we call this nodebased product-flow (Node-BPF) approach. With this approach the formulation 
keeps track of the quantity of the products at the nodes (entering to and/or leaving from the nodes), rather than in between the nodes.

In order to demonstrate the proposed approach we reformulate a 4-index Arc-BPF LRP model from the literature by considering Node-BPF approach. This approach enables us to develop a 3-index Node-BPF LRP model with fewer constraints and variables. It is shown that the Node-BPF model outperforms the Arc-BPF model in terms of both optimality gap and objective function value in a given time. Hence, the Node-BPF approach we introduce in this study is a promising attempt to improve the computational efficiency of the LRP models purely through modeling.

After developing a computationally more efficient model we then develop a 3-phase clustering-based model specific heuristic to shorten the solution time. The heuristic leads to promising results showing that it can be adopted as a decision support tool to solve the real life problems in a reasonable amount of time.

We claim that the proposed Node-BPF formulation approach offers a valid alternative to the widely used Arc-BPF approach. It can be applied to existing and future LRP models to potentially improve the solution time.

Although the model studied in this paper may seem to be derived for the specific military logistics problem, we strongly believe that it can be applied to a wide variety of distribution systems after some straightforward modifications. One such possible application area is Humanitarian Relief Logistics where the main objectives are to predict demands, identify priorities, sort supplies, coordinate supply chain and direct the transportation and distribution in order to deliver relief to people affected by a disaster.

Since our model contains most of the real world aspects and offers a relatively better computational efficiency with the proposed formulation approach, we hope that it will help model complex logistics systems more realistically.

Acknowledgements The authors are grateful to Location Area Editor and two anonymous referees for providing constructive comments and suggestions that improved the quality of the paper.

\section{References}

Albareda-Sambola M, Díaz JA, Fernández E (2005) A compact model and tight bounds for a combined location-routing problem. Comput Oper Res 32:407-428

Ambrosino D, Scutellà MG (2005) Distribution network design: new problems and related models. Eur J Oper Res 165:610-624

Baldacci R, Mingozzi A, Calvo RW (2011) An exact method for the capacitated location-routing problem. Oper Res 59(5):1284-1296

Barreto S, Ferreira C, Paixao J, Santos BS (2007) Using clustering analysis in a capacitated location-routing problem. Eur J Oper Res 179:968-977

Belenguer JM, Benavent E, Prins C, Prodhon C, Calvo RW (2011) A branch-and-cut method for the capacitated location-routing problem. Comput Oper Res 38:931-941 
Berman O, Jaillet P, Simchi-Levi D (1995) Location-routing problems with uncertainty. In: Drezner Z (ed) Facility location a survey of applications and methods. Springer, New York, pp 427-452

Billionnet A, Elloumi S, Djerbi LG (2005) Designing radio-mobile access networks based on synchronous digital hierarchy rings. Comput Oper Res 32:379-394

Bozkaya B, Yanik S, Balcisoy S (2010) A GIS-based optimization framework for competitive multi-facility location-routing problem. Netw Spat Econ 10:297-320

Braysy O, Gendreau M (2005) Vehicle routing problem with time windows, part i: route construction and local search algorithms. Transp Sci 39:104-118

Bruns A, Klose A (1995) An iterative heuristic for location-routing problems based on clustering. In: Proceedings of the second international workshop on distribution logistics, Oegstgeest, The Netherlands

Crainic TG, Laporte G (1997) Planning models for freight transportation. Eur J Oper Res 97:409_ 438

Duhamel C, Lacomme P, Prins C, Prodhon C (2010) A GRASPxELS approach for the capacitated location-routing problem. Comput Oper Res 37:1912-1923

Hansen PH, Hegedahl B, Hjortkjaer S, Opel B (1994) A heuristic solution to the warehouse location-routing problem. Eur J Oper Res 76:111-127

Karaoglan I, Altiparmak F, Kara I, Dengiz B (2011) A branch and cut algorithm for the locationrouting problem with simultaneous pickup and delivery. Eur J Oper Res 211:318-332

Laporte G (1988) Location-routing problems. In: Golden BL, Asad A (eds) Vehicle routing: methods and studies. North-Holland Publishing, Amsterdam, Holland, pp 163-198

Laporte G, Dejax PJ (1989) Dynamic location-routing problems. J Oper Res Soc 40(5):471-482

Laporte G, Nobert Y (1981) An exact algorithm for minimizing routing and operating costs in depot location. Eur J Oper Res 6:224-226

Laporte G, Nobert Y, Arpin D (1986) An exact algorithm for solving a capacitated locationrouting problem. Ann Oper Res 6:293-310

Laporte G, Nobert Y, Taillefer S (1988) Solving a family of multi-depot vehicle routing and location-routing problems. Transp Sci 22(3):161-172

List GF, Mirchandani PB, Turnquist MA, Zografos KG (1991) Modeling and analysis for hazardous materials transportation: Risk analysis, routing/scheduling and facility location. Transp Sci 25(2):100-114

Melechovsky J, Prins C, Calvo RW (2005) A metaheuristic to solve location-routing problem with non-linear costs. Journal of Heuristics 11:375-391

Miller CE, Tucker AW, Zemlin RA (1960) Integer programming formulation of traveling salesman problems. J Assoc Comput Mach 7:326-329

Min H (1996) Consolidation terminal location-allocation and consolidated routing problems. J Bus Logist 17(2):235-263

Min H, Jayaraman V, Srivastava R (1998) Combined location-routing problems: a synthesis and future research directions. Eur J Oper Res 108:1-15

Nagy G, Salhi S (1996) A nested location-routing heuristic using route length estimation. Stud Locat Anal 10:109-127

Nagy G, Salhi S (2007) Location-routing: issues, models and methods. Eur J Oper Res 177:649_ 672

Nambiar JM, Gelders LF, Wassenhove LNV (1989) Plant location and vehicle routing in the Malaysian rubber smallholder sector: a case study. Eur J Oper Res 38:14-26

Or I, Pierskalla WP (1979) A transportation location-allocation model for regional blood banking. AIIE Trans 11(2):86-95

Perl J, Daskin MS (1985) A warehouse location-routing problem. Transp Res B 19B(5):381-396

Prins C, Prodhon C, Soriano P, Ruiz A, Calvo RW (2007) Solving the capacitated location-routing problem by a cooperative Lagrangean relaxation-granular tabu search heuristic. Transp Sci 41(4):470-483

Salhi S, Fraser M (1996) An integrated heuristic approach for the combined location vehicle fleet mix problem. Stud Locat Anal 8:3-21

Salhi S, Rand GK (1989) The effect of ignoring routes when locating depots. Eur J Oper Res 39:150-156 
Schwardt M, Dethloff J (2005) Solving a continuous location-routing problem by use of a selforganizing map. Int J Phys Distrib Logist Manag 35(6):390-408

Solomon MM (1986) On the worst-case performance of some heuristics for the vehicle routing and scheduling problem with time window constraints. Networks 16:161-174

Solomon MM (1987) Algorithms for the vehicle routing and scheduling problems with time window constraints. Oper Res 35:254-265

Srivastava R, Benton WC (1990) The location-routing problem: considerations in physical distribution system design. Comput Oper Res 17(5):427-435

Tavakkoli-Moghaddam R, Makui A, Mazloomi Z (2010) A new integrated mathematical model for a bi-objective multi-depot location-routing problem solved by a multi-objective scatter search algorithm. J Manuf Syst 29:111-119

Toyoglu H, Karasan OE, Kara BY (2011) Distribution network design on the battlefield. Nav Res Logist 58(3):188-209

Wu T, Low C, Bai J (2002) Heuristic solutions to multi-depot location-routing problems. Comput Oper Res 29:1393-1415

Yi W, Ozdamar L (2007) A dynamic logistics coordination model for evacuation and support in disaster response activities. Eur J Oper Res 179:1177-1193 